\title{
Yield strength dependence on strain rate of molybdenum-alloy nanofibers
}

\author{
P. E. Loya,${ }^{1}$ Y. Z. Xia, ${ }^{2}$ C. Peng,${ }^{1}$ H. Bei, ${ }^{3}$ P. Zhang, ${ }^{1}$ J. Zhang, ${ }^{1}$ E. P. George, ${ }^{2,3}$ Y. F. Gao, ${ }^{2,3}$ \\ and J. Lou ${ }^{1}$ \\ ${ }^{1}$ Department of Materials Science and NanoEngineering, Rice University, Houston, Texas 77005, USA \\ ${ }^{2}$ Department of Materials Science and Engineering, University of Tennessee, Knoxville, \\ Tennessee 37996, USA \\ ${ }^{3}$ Materials Science and Technology Division, Oak Ridge National Laboratory, Oak Ridge, \\ Tennessee 37831, USA
}

(Received 24 April 2014; accepted 15 June 2014; published online 25 June 2014)

\begin{abstract}
The yield strength dependence on strain rate was studied for molybdenum-alloy nanofibers with varying initial dislocation density at three different pre-strain levels. In-situ tensile experiments at three displacement rates were carried out in a scanning electron microscope. Yield strength and its scatter decreased as a function of the pre-strain level for different displacement rates. A statistical model was used to analyze the results, and a negative strain rate dependence was inferred from the yield experiments. This finding suggests the need for theoretical investigations since classical models such as dynamic strain aging may have limitations at such nanoscales. (c) 2014 AIP Publishing LLC.

[http://dx.doi.org/10.1063/1.4885377]
\end{abstract}

The "smaller is stronger" phenomenon arises from observations that nanoscale materials can have anomalously high strength. ${ }^{1,2}$ More recently, an understanding of the underlying mechanism has emerged. ${ }^{3,4}$ The dislocations present in the material before testing, as well as those generated during the mechanical testing process, can be annihilated by the large amount of free surface present in these samples. ${ }^{3}$ The stresses required to change the configuration of small numbers of dislocations or to nucleate new dislocations are larger than those for continuum slip that involves a huge number of dislocations. In addition, the stressed volumes in different specimens may sample different numbers of dislocations or various dislocation configurations. A commonly observed phenomenon at macroscopic scales is the rate dependence of yield strength, with origins closely related to forest dislocation hardening, diffusion of solute atoms near dislocations, or other thermally activated dislocation-related mechanisms. Clearly, when specimen size decreases, the discrete nature and stochastic behavior of dislocation plasticity is anticipated to emerge, with a complex convolution of size-dependent strength, spatial statistics, and strain rate sensitivity.

By utilizing nano-indentation and focused ion beam (FIB) milling, e.g., Refs. 1-4, it is now possible to explore size and rate dependence of plasticity in a wide variety of materials. Schneider et al. ${ }^{5}$ explored the strain rate sensitivity of Mo in various crystalline orientations. They were able to examine mechanical behaviors of FIB produced pure Mo pillars using nano-compression with a nano-indenter. ${ }^{5}$ These experiments showed that over a wide range of strain rates $\left(10^{-6} \mathrm{~s}^{-1}-10^{-2} \mathrm{~s}^{-1}\right),\langle 001\rangle$ Mo exhibited increased strain rate sensitivity with size. ${ }^{5}$ Here, we report our ratedependency investigation of Mo fibers that differ significantly from previous work in the following two respects. First, we use directionally solidified fibers that are free of FIB-induced damage $^{6}$ that can cause surface hardening. ${ }^{7-9}$ Second, we examine nanofibers under tensile loading conditions, which are an order of magnitude or more, longer than those used in compression tests. Thus, the complex interactions between strain-rate sensitivity and spatial statistics of finding preexisting defects can be explored and quantitatively tuned by changing the pre-strain levels and sample lengths.

In this work, we investigate the strain rate sensitivity of body centered cubic $\langle 100\rangle$ Mo-alloy nanofibers with varying dislocation densities utilizing in-situ tensile tests in a scanning electron microscope (SEM). By combining a quantitative inSEM nanoindenter, e.g., Refs. 9 and 10, and a micromechanical device, ${ }^{1-15}$ with a sample preparation method that avoided surface modifications due to FIB milling, ${ }^{6}$ the mechanical properties of Mo-alloy nanofibers with different initial dislocation densities were systematically investigated at different displacement rates. The average yield strength and its scatter were found to generally decrease with increasing pre-strain level at all three displacement rates. Finally, the strain rate sensitivity was calculated, and a negative strain rate sensitivity was obtained for the displacement range tested. This opens up opportunities for modeling and simulation to investigate the origin of this negative strain rate sensitivity at nanoscales.

Pristine, low defect density Mo-alloy nanofibers in a NiAl-Mo eutectic system ${ }^{16}$ were synthesized by directional solidification. ${ }^{17}$ These nanofibers are relatively long with a chemical composition of $86 \mathrm{Mo}-10 \mathrm{Al}-4 \mathrm{Ni}$ (at. \%) and are embedded in a 55Ni-45Al (at. \%) matrix. ${ }^{16}$ The directionally solidified NiAl/Mo composite was cut into small discs with a diameter of $6.5 \mathrm{~mm}$ and a thickness of $2 \mathrm{~mm}$. These discs were subjected to a compressive pre-straining process that allows for the dislocation densities of the nanofibers to be tuned depending on the amount of the pre-strain. ${ }^{17,18}$ The pre-strain levels that were chosen in this investigation were $0 \%, 4 \%$, and $16 \%$.

A clear advantage of the $\mathrm{NiAl} / \mathrm{Mo}$ composite is that the nanofibers can be easily extracted from the NiAl matrix without inducing damage. $\mathrm{NiAl}$ can be etched away by an acidic solution whereas Mo can be etched in a basic solution; therefore, selective etching is easily achievable. In our process, the $\mathrm{NiAl}$ matrix was etched away at a rate of $1 \mu \mathrm{m}$ per 
minute using an $80 \mathrm{H}_{2} \mathrm{O}-10 \mathrm{HCl}-10 \mathrm{H}_{2} \mathrm{O}_{2}$ (vol. \%) solution leaving behind exposed Mo-alloy nanofibers. The fibers were then sonicated in order to break and separate them from the residual matrix and dispersed on a titanium-coated silicon wafer for subsequent manipulations onto a micromechanical testing platform. ${ }^{11-15}$ Single crystal Mo-alloy nanofibers that were tested in this study all have a $\langle 100\rangle$ growth direction as shown by the example in Figure 1(a). The dislocation densities in these nanofibers at several different pre-strain levels were carefully measured by Phani et al. ${ }^{18}$ using STEM imaging techniques. In each case, dislocation densities were measured over a large length of the nanofiber to get a good statistical representation of dislocation densities, and to check the uniformity of those densities throughout the length of the nanofiber. As-grown (or 0\% pre-strain) nanofibers contained, on average, one line defect per $37 \mu \mathrm{m}$ of fiber length. ${ }^{18} 4 \%$ pre-strain fibers contained a dislocation density of approximately $1.8-8.2 \times 10^{12} \mathrm{~m}^{-2}$, but the densities were not uniform along the fiber axis with areas of both low and high densities present. ${ }^{18}$ Finally, 16\% pre-strain fibers contained a uniform distribution of dislocations with densities of about $4.4 \times 10^{13} \mathrm{~m}^{-2} .^{18}$

In-situ tensile tests were conducted in an FEI Quanta 400 FEG ESEM using an Agilent G200 InSEM Nano-indenter. The nano-indenter was used to record force/displacement data from the experiments and to actuate a micro-mechanical device, ${ }^{6-10}$ shown in Figure 1(b), which was used to produce a uniaxial tensile force on the nanofiber samples. In order to mount the sample on the micro-mechanical device, a small amount of epoxy was placed on each shuttle and the dispersed nanofibers were manipulated into testing positions using an optical probe station (Micromanipulator, Inc.). Once the nanofibers were placed on the device, they were transported to an FEI Strata 235 dual beam FIB where the nanofibers were welded in place with platinum deposited through ion

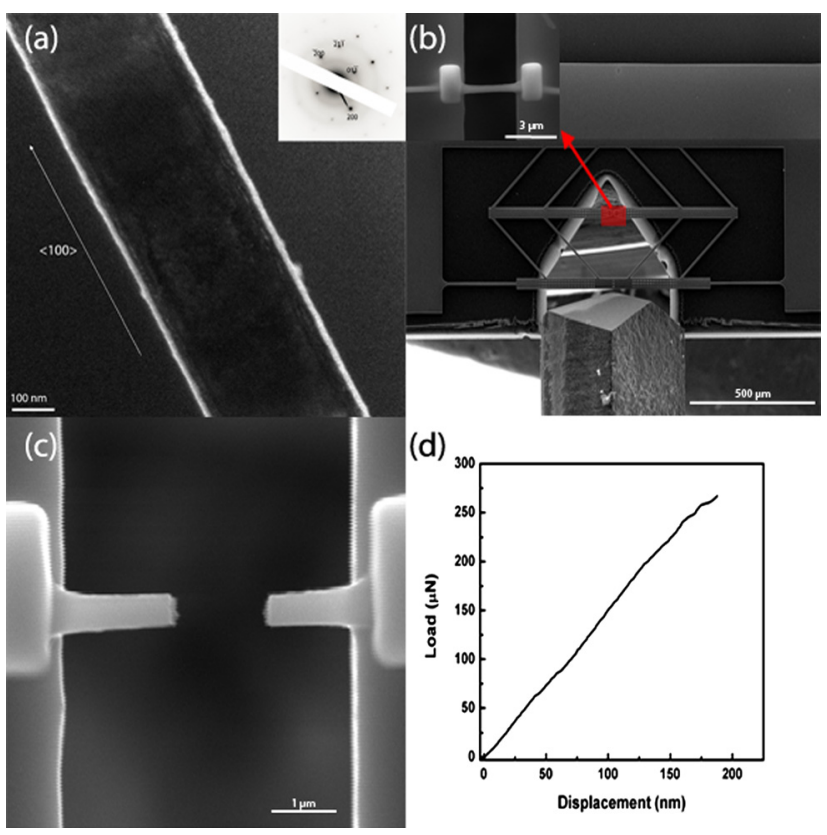

FIG. 1. (a) Dark field TEM image of Mo fiber showing $\langle 100\rangle$ growth direction. (b) SEM image of in-situ set up. (c) SEM image of fractured Mo fiber. (d) Corresponding load-displacement curve of sample in (c). beam induced deposition. Each nanofiber/microdevice assembly was mounted on an SEM stub and secured with silver epoxy. Once inside the FEI Quanta SEM chamber, a series of images was taken in order to measure the gauge length and diameter of each fiber. The gauge length varied for different samples so measurements of clamping points spacing were carried out for each fiber. Samples from each of the three prestrain levels were tested at three displacement rates: $20 \mathrm{~nm} / \mathrm{s}$, $25 \mathrm{~nm} / \mathrm{s}$, and $100 \mathrm{~nm} / \mathrm{s}$, which are in the range accessible to our in-situ set up. It is recognized that in order to further this study, future experiments looking into a much broader range of displacement rates need to be carried out.

The mechanical responses of the Mo alloy nanofibers tested under different displacement rates were analyzed for each of the three pre-strain levels. A representative SEM image of a nanofiber fractured under tension is shown in Figure 1(c). Localized plastic deformation close to the fracture surfaces is clearly observed; this feature was consistently observed in many of our tested samples. Most samples also exhibited load-displacement curves that are typical for a brittle material as shown in Figure 1(d). Using the measured sample dimensions, corresponding stress-strain curves could be determined. It should be noted that evidence of plastic deformation has been seen in similar nanofibers even when, macroscopically, they appear to fracture in a brittle manner. Chisholm et al. ${ }^{19}$ showed that dislocations are mobile within the sample volume even in the presumed "elastic" loading regime. It should be noted that in our stress calculations the cross-section of each nanofiber was assumed be round. The actual cross-section shape varied from fiber to fiber: In some instances, it was more circular while in others it had a more square-like shape with rounded corners. An accurate measurement of the actual cross-section area was very challenging in our testing set up. Therefore, the measured "diameter" value could be the diagonal or the side of the square crosssection, or the real diameter of the nanofiber if it was actually circular as assumed. As such, the actual cross sectional area may be off by as much as $\pm 20 \%$, and this could lead to variations in the reported yield strength values. ${ }^{14}$ Presumably, however, these are random and not systematic errors.

As shown in Figure 2, it is clear that, as the pre-strain level, i.e., the dislocation density, increases, both the average values of yield strength and their scatter decrease. Johanns et $a .^{20}$ performed uni-axial tensile experiments on similar nanofibers and reported reduced yield strength and scatter as a function of the increasing pre-strain level. Our experiments show this trend for the lower displacement rates $(20 \mathrm{~nm} / \mathrm{s}$ and $25 \mathrm{~nm} / \mathrm{s}$ ), which is more comparable to their displacement rate. This result is also consistent with the general trend reported by Bei et al. ${ }^{17}$ in uni-axial compression tests performed on Mo alloy nanopillars.

However, the above trend is not evident at the higher displacement rate of $100 \mathrm{~nm} / \mathrm{s}$ as shown in Figure 2(c). At this much higher displacement rate, the statistical differences in the yield stress for pre-strain levels of 0 and $4 \%$ are not as obvious as for samples tested at the two lower displacement rates described previously. Additionally, the scatter in the data for the $16 \%$ pre-strain specimens is lower at $100 \mathrm{~nm} / \mathrm{s}$ than at the slower displacement rates. Therefore, 
(a)
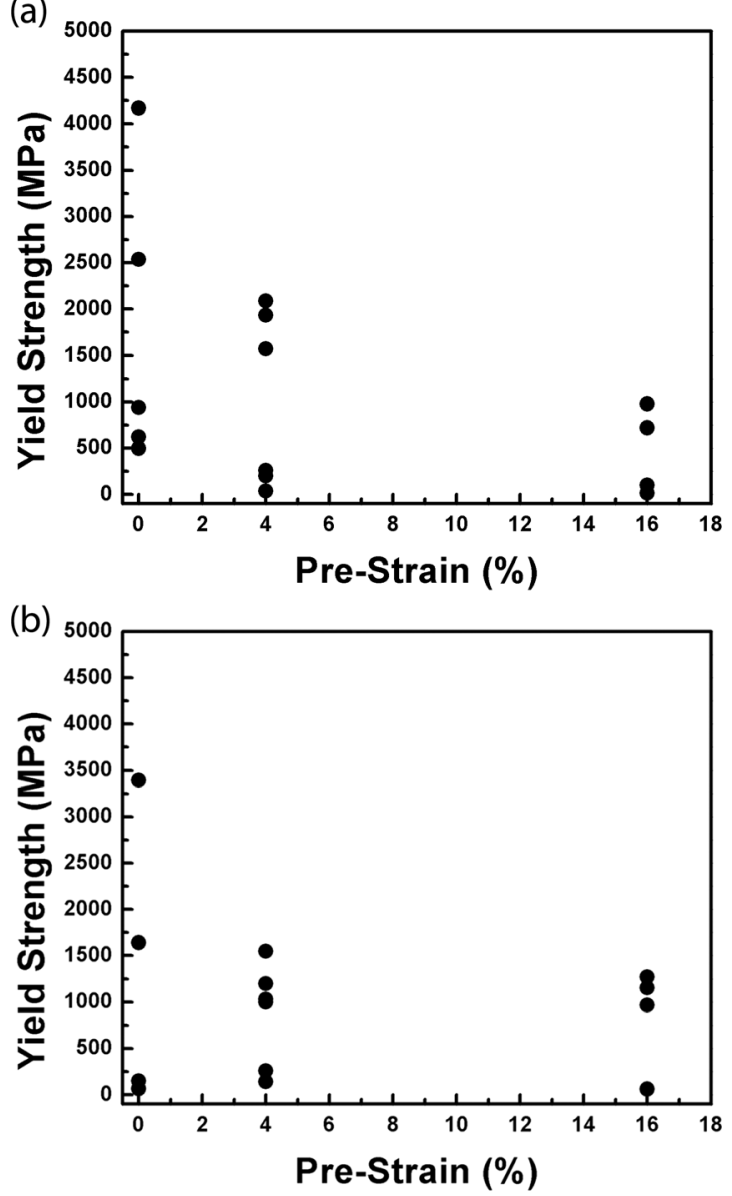

(c)

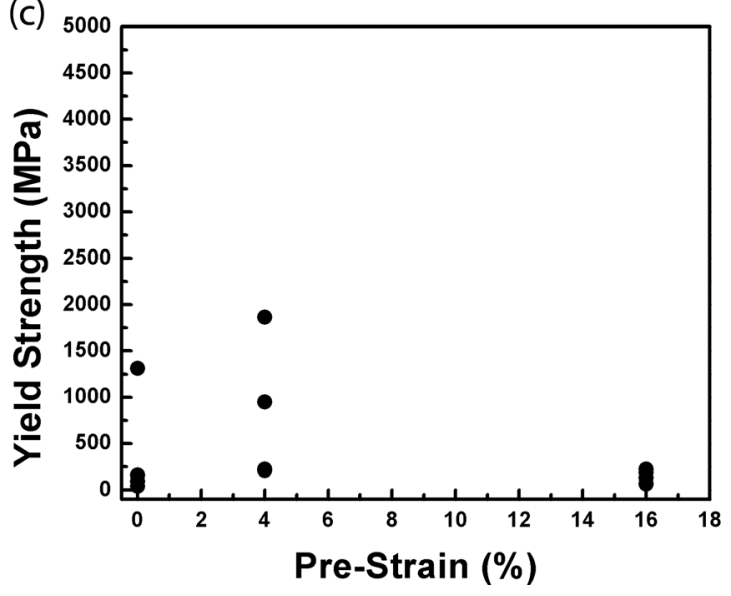

FIG. 2. Yield strength vs. pre-strain for (a) $20 \mathrm{~nm} / \mathrm{s}$, (b) $25 \mathrm{~nm} / \mathrm{s}$, and (c) $100 \mathrm{~nm} / \mathrm{s}$ displacement rate.

displacement/strain rate plays an important role in the deformation behaviors of these Mo-alloy nanofibers.

A minimum of four samples were tested for each prestrain level at each of the three displacement rates. Our yield stress values correlate well with the experiments done on similar samples by Johanns et al. ${ }^{20}$ Results are shown to be stochastic; however, these experiments are stochastic in nature even when numerous samples are tested. ${ }^{20}$ That is, the sample size is comparable to the feature sizes of dislocation microstructure, so that the deformation behavior is stochastic. It is the objective of our work to de-convolute the coupling of this stochastic behavior and strain rate sensitivity, with the aid of the statistic model presented later in this paper.
It is interesting that increasing the pre-strain level (i.e., dislocation density) or the strain/displacement rate have similar effects on reducing the yield strength and its scatter. As the displacement rate is increased by a factor of five, the average yield strength of all the specimens approaches that of the $16 \%$ pre-strain sample, and even approaches the bulk value for samples with the highest pre-strain level tested (Fig. 2(c)). ${ }^{17,20}$ In order to understand this phenomenon of decreasing scatter as the pre-strain level is increased, ${ }^{17,20}$ a statistical model can be adopted. The deformation of defectfree nanofibers is assumed to be governed by homogeneous nucleation of dislocations, so that the strength approaches the theoretical value $\sigma_{t h}$. On the other hand, the yielding behavior may be governed by the activation of a pre-existing dislocation, in which case the critical resolved shear stress $\tau_{\text {CRSS }}$ provides a lower-limit bulk behavior. ${ }^{21}$ Phani et al. ${ }^{22}$ developed the statistical model considering a random spatial distribution and orientation of dislocations within the gauge length tested. If the gauge length did not have any preexisting dislocations, then the yield strength should approach the theoretical value because dislocations would have to be nucleated in order for yielding to occur. However, none of our samples had yield strengths approaching the theoretical value at any of the pre-strain levels; therefore, it is reasonable to believe that dislocations are present within the tested gauge lengths. The model uses a weakest-link approach, considering the relationship between a random Schmid factor $(s)$ and the yield strength, to determine the critical resolved shear stress $\left(\tau_{c r s s}\right)$ or the bulk strength $\left(\sigma_{b}=\tau_{c r s s} / s_{\max }\right)$ given a gauge length $l$ and pre-existing dislocation density $\rho_{1 D} \cdot{ }^{22} s_{\max }$ and $s_{\min }$ represent the maximum and minimum Schmid factors which are 0.5 and 0.0 , respectively, in our analysis. The model essentially provides a spatial sampling of the weakest defect that leads to the material yield, so the predicted scatter manifests the convolution of strength and spatial distribution of pre-existing defects.

Figure 3(a) shows that the strengths (both the upper bound (95\% probability) and lower bound (5\% probability)) are the theoretical strength when the product of the specimen length and defect density, $\rho_{1 D} l=0$, while the contribution of the bulk strength increases as $\rho_{1 D}$ increases. The following equations can be used to predict the upper $\sigma_{U B}$ and lower bounds $\sigma_{L B}$,

$$
\begin{gathered}
\sigma_{U B}=\sigma_{t h} \text { for } \rho_{1 D} l \leq \frac{\ln \alpha}{\frac{s_{\min }}{s_{\text {max }}}-1} \\
\sigma_{U B}=\sigma_{b}\left(\frac{\rho_{1 D} l}{\rho_{1 D} l+\ln \alpha}\right) \text { for } \rho_{1 D} l>\frac{\ln \alpha}{\frac{s_{\min }}{s_{\max }}-1}, \\
\sigma_{L B}=\sigma_{t h} \text { for } \rho_{1 D} l \leq \frac{\ln (1-\alpha)}{\frac{s_{\min }}{s_{\max }}-1} \\
\sigma_{L B}=\sigma_{b}\left(\frac{\rho_{1 D} l}{\rho_{1 D} l+\ln (1-\alpha)}\right) \text { for } \rho_{1 D} l>\frac{\ln (1-\alpha)}{\frac{s_{\min }}{s_{\max }}-1}
\end{gathered}
$$

where $\alpha$ is the strength probability of lower bound. These four equations allow us to generate the bounds plotted in Figure 3(a). 

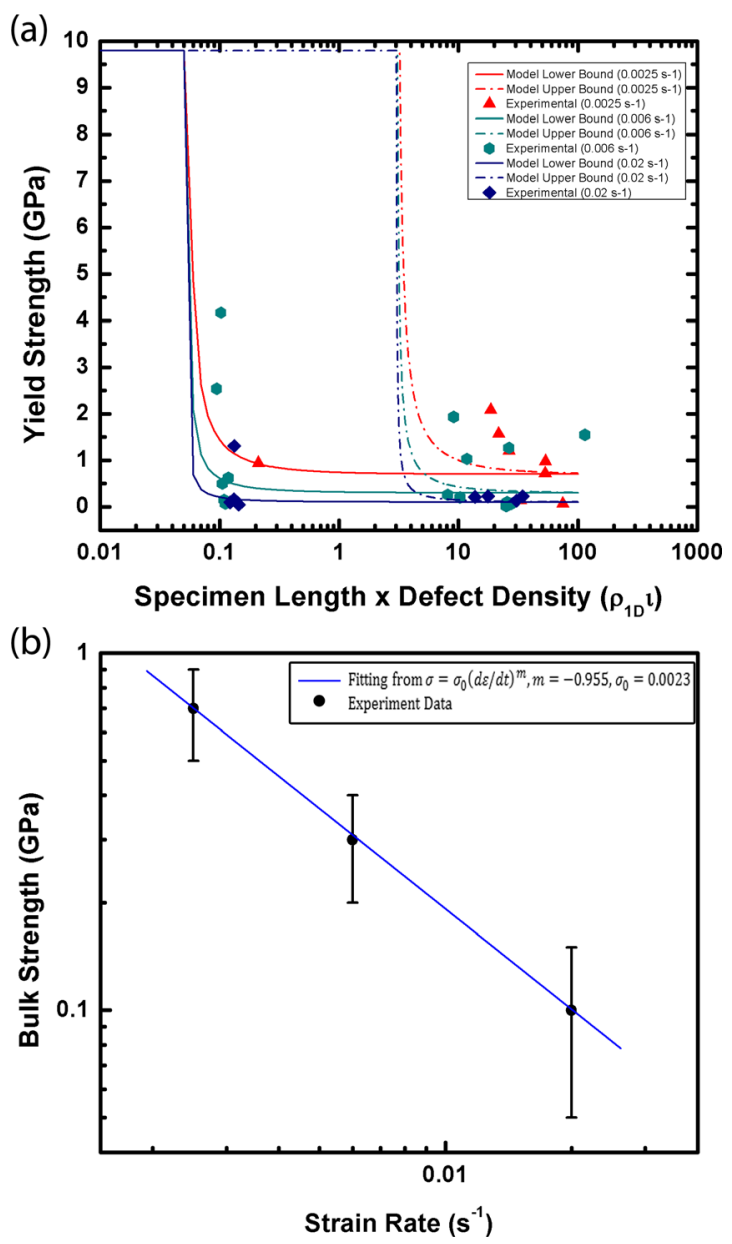

FIG. 3. (a) Model prediction and experimental comparison of yield strength, and (b) dependence of bulk strength on strain rate fitted strain rate sensitivity parameter.

Most of the experimental data are located near the bulk strength, presumably because of the relatively high strain rate applied in our experiment and the relatively high probability of dislocations being present. That is, the probability of having dislocations in the gauge length with a $\tau_{\mathrm{CRSS}} / \mathrm{s}$ equal to or close to that of the bulk strength is relatively high. $^{21,22}$ The fitted bulk strengths are $0.6-0.9 \mathrm{GPa}$, $0.2-0.4 \mathrm{GPa}$, and $0.05-0.2 \mathrm{GPa}$ for strain rates of $\sim 0.0025$ $\mathrm{s}^{-1}, \sim 0.006 \mathrm{~s}^{-1}$, and $\sim 0.02 \mathrm{~s}^{-1}$, respectively. These values show that the bulk strength decreases as a function of strain rate, which implies that the pre-existing defects can be activated at lower tensile stresses and that heterogeneous yielding occurs more easily when the fiber is loaded at higher strain rates. Therefore, negative strain rate sensitivity would be expected. One important parameter, the strain rate sensitivity, $m$, is defined in terms of an empirical fitting formula

$$
m=\frac{d(\ln \sigma)}{d(\ln \dot{\varepsilon})},
$$

which can be determined by fitting the stress-strain curves in logarithmic scales at different strain rates. As shown in Figure 3(b), a negative strain rate sensitivity of $m=-0.955$ is obtained for our nanofibers, which is different with the reported strain rate sensitivity $(\mathrm{m}=0.085)$ of bulk Mo. ${ }^{5}$ However, it is important to note that the negative strain rate sensitivity is observed here in a relatively narrow strain-rate range that is limited by our instrument capability. There is a possibility that the strain rate sensitivity might deviate from these values when a wider strain rate range is achieved as seen in Schneider et al. ${ }^{5}$

In summary, we utilized a micro-mechanical testing platform in combination with a nano-indenter inside a SEM to investigate the strain rate dependent mechanical behaviors of Mo alloy nanofibers with varying dislocation densities. We have shown a trend of decreasing yield strength and data scatter as a function of the pre-strain level for samples tested at lower displacement rates, which are comparable to previously reported results. ${ }^{17,20}$ When the strain rate is increased by a factor of eight, the yield strength decreases for all prestrain levels. A negative strain rate sensitivity was observed over the range of tested displacement rates. The complex coupling between stochastic deformation behavior (as shown by the data scatter in Fig. 2) and negative strain rate sensitivity has been deconvoluted by our statistical model in Fig. 3(a), without which the large data scatter does not permit a clear view of the rate dependency. The model, which is based on the weakest link, suggests that the probability of finding a pre-existing defect with the highest Schmid factor should be superposed with any mechanisms that are responsible for the rate dependency. However, the mechanisms responsible for the negative strain sensitivity cannot be obtained from this study due to the lack of microstructural characterization. Nonetheless, these findings provide opportunities for atomistic simulations, which are capable of modeling systems of similar sizes.

The authors acknowledge the support from NSF Grant Nos. DMR-1128818 and CMMI-0928297, and the Welch Foundation Grant No. C-1716 (P.E.L., C.P., P.Z., J.Z., and J.L.), and the U.S. Department of Energy, Basic Energy Sciences, Materials Sciences, and Engineering Division (Y.Z.X., H.B., E.P.G., and Y.F.G.).

${ }^{1}$ M. D. Uchic, D. M. Dimiduk, J. N. Florando, and W. D. Nix, Science 305, 986 (2004).

${ }^{2}$ M. D. Uchic and D. M. Dimiduk, Mater. Sci. Eng., A 400, 268 (2005).

${ }^{3}$ J. R. Greer, W. C. Oliver, and W. D. Nix, Acta Mater. 53, 1821 (2005).

${ }^{4}$ J. Y. Kim and J. R. Greer, Appl. Phys. Lett. 93, 101916 (2008).

${ }^{5}$ A. S. Schneider, B. G. Clark, C. P. Frick, P. A. Gruber, and E. Arzt, Mater. Sci. Eng., A 508, 241 (2009).

${ }^{6}$ H. Bei, S. Shim, E. P. George, M. K. Miller, E. G. Herbert, and G. M. Pharr, Scr. Mater. 57, 397 (2007).

${ }^{7}$ H. Bei, S. Shim, M. K. Miller, G. M. Pharr, and E. P. George, Appl. Phys. Lett. 91, 111915 (2007).

${ }^{8}$ D. Kiener, C. Motz, M. Rester, M. Jenko, and G. Dehm, Mater. Sci. Eng., A 459, 262 (2007).

${ }^{9}$ D. S. Gianola, A. Sedlmayr, R. Monig, C. A. Volkert, R. C. Major, E. Cyrankowski, S. A. S. Asif, O. L. Warren, and O. Kraft, Rev. Sci. Instrum. 82, 063901 (2011).

${ }^{10}$ J. Y. Kim, D. Jang, and J. R. Greer, Acta Mater. 58, 2355 (2010).

${ }^{11}$ Y. Ganesan, Y. Lu, C. Peng, H. Lu, R. Ballarini, and J. Lou, J. Microelectromech. Syst. 19, 675 (2010).

${ }^{12}$ C. Peng, Y. Zhan, and J. Lou, Small 8, 1889 (2012).

${ }^{13}$ C. Peng, Y. Ganesan, Y. Lu, and J. Lou, J. Appl. Phys. 111, 063524 (2012).

${ }^{14}$ Y. Lu, C. Peng, Y. Ganesan, J. Huang, and J. Lou, Nanotechnology 22, 355702 (2011).

${ }^{15}$ C. Peng, Y. Zhong, Y. Lu, S. Narayanan, T. Zhu, and J. Lou, Appl. Phys. Lett. 102, 083102 (2013).

${ }^{16}$ H. Bei and E. P. George, Acta Mater. 53, 69 (2005). 
${ }^{17}$ H. Bei, S. Shim, G. M. Pharr, and E. P. George, Acta Mater. 56, 4762 (2008).

${ }^{18}$ P. S. Phani, K. E. Johanns, G. Duscher, A. Gali, E. P. George, and G. M. Pharr, Acta Mater. 59, 2172 (2011).

${ }^{19}$ C. Chisholm, H. Bei, M. B. Lowry, J. Oh, S. A. S. Asif, O. L. Warren, Z. W. Shan, E. P. George, and A. M. Minor, Acta Mater. 60, 2258 (2012).
${ }^{20}$ K. E. Johanns, A. Sedlmayr, P. S. Phani, R. Monig, O. Kraft, E. P. George, and G. M. Pharr, J. Mater. Res. 27, 508 (2012).

${ }^{21}$ T. L. Li, H. Bei, J. R. Morris, E. P. George, and Y. F. Gao, Mater. Sci. Technol. 28, 1055 (2012).

${ }^{22}$ P. S. Phani, K. E. Johanns, E. P. George, and G. M. Pharr, Acta Mater. 61, 2489 (2013). 